\title{
THE NUMERICAL INDEX OF NONSELFADJOINT OPERATOR ALGEBRAS
}

\author{
YOSHIHIRO NAKAMURA, KICHI-SUKE SAITO, AND KAZUNARI SAKABA
}

(Communicated by Palle E. T. Jorgensen)

\begin{abstract}
Let $\mathfrak{A}$ be a (not necessarily selfadjoint) closed subalgebra of $B(H)$ that is the algebra of all bounded linear operators on a Hilbert space $H$. In this note, we prove that the range of numerical index of $\mathfrak{A}$ as an algebra is the whole of the interval $\left[\frac{1}{2}, 1\right]$.
\end{abstract}

Let $H$ be a complex Hilbert space and $B(H)$ the algebra of all bounded linear operators on $H$. Given $T \in B(H)$, we define the numerical range $W(T)$ and the numerical radius $w(T)$ as

$$
\begin{aligned}
W(T) & =\{(T x, x): x \in H,\|x\|=1\}, \\
w(T) & =\sup \{|\lambda|: \lambda \in W(T)\} .
\end{aligned}
$$

Let $\mathfrak{A}$ be a closed (not necessarily selfadjoint) subalgebra of $B(H)$. As in [1, 2], we recall the numerical index $n(\mathfrak{A})$ of $\mathfrak{A}$ is defined by

$$
n(\mathfrak{A})=\inf \{w(T): T \in \mathfrak{A},\|T\|=1\} .
$$

Since $\frac{1}{2}\|T\| \leq w(T) \leq\|T\|$ for every $T \in B(H)$, we have $\frac{1}{2} \leq n(\mathfrak{A}) \leq 1$. As in [3], Crabb, Duncan, and McGregor showed that if $\mathfrak{A}$ is selfadjoint, that is, $\mathfrak{A}$ is a $C^{*}$-algebra, then $n(\mathfrak{A})=1$ or $\frac{1}{2}$ if $\mathfrak{A}$ is commutative or not commutative, respectively. In this note, we shall study the numerical index of nonselfadjoint operator algebras. As a result, we shall prove that the range of numerical index of nonselfadjoint operator algebras is the whole of the closed interval $\left[\frac{1}{2}, 1\right]$. That is, we have the following theorem.

Theorem. For every real number $r$ such that $\frac{1}{2} \leq r \leq 1$, there exists a Hilbert space $H$ and $a$ (nonselfadjoint) closed subalgebra $\mathfrak{A}$ of $B(H)$ such that $n(\mathfrak{A})=$ $r$.

Proof. Let $r$ be a real number such that $\frac{1}{2} \leq r \leq 1$. Let $A=\left[\begin{array}{ll}0 & 0 \\ 1 & 0\end{array}\right]$ and let $B$ be a normal operator on a Hilbert space $K$ such that the spectrum $\sigma(B)$ of $B$ is $\{\lambda \in \mathbb{C}:|\lambda|=r\}$. Put $T=A \oplus B$ on $H=\mathbb{C}^{2} \oplus K$. We now consider the closed subalgebra $\mathfrak{A}_{T}$ generated by $T$ and $I$, that is, $\mathfrak{A}_{T}$ is the norm closure

Received by the editors August 1, 1991.

1991 Mathematics Subject Classification. Primary 47D25, 46L05; Secondary 47A12.

Key words and phrases. Operator algebra, numerical index.

Research partially supported by a Grant-in-Aid for Scientific Research from the Japanese Ministry of Education. 
of $\{p(T): p$ is polynomial $\}$. Since $T$ is the convexoid operator such that $\|T\|=1$ and $w(T)=r$, we have $n\left(\mathfrak{A}_{T}\right) \leq r$. Therefore, to prove $n\left(\mathfrak{A}_{T}\right) \geq r$, it is sufficient to prove that $w(p(T)) \geq r\|p(T)\|$ for every polynomial $p$, by the continuity of numerical radius.

For every polynomial $p$, we now have $w(p(T))=\max \{w(p(A)), w(p(B))\}$, $\|p(T)\|=\max \{\|p(A)\|,\|p(B)\|\}$, and $w(p(B))=\|p(B)\|=\max _{|z|=r}|p(z)|$. Putting $p(z)=\sum_{n=0}^{m} a_{n} z^{n}$, it is clear that

$$
w(p(A))=w\left(a_{0} I+a_{1} A\right)=\left|a_{0}\right|+\frac{1}{2}\left|a_{1}\right|
$$

and

$$
\|p(A)\|=\left\|\left[\begin{array}{cc}
a_{0} & 0 \\
a_{1} & a_{0}
\end{array}\right]\right\|=\left(\left|a_{0}\right|^{2}+\frac{1}{4}\left|a_{1}\right|^{2}\right)^{1 / 2}+\frac{1}{2}\left|a_{1}\right| .
$$

If $\|p(A)\| \leq\|p(B)\|$ then $w(p(A)) \leq\|p(B)\|$, and so $w(p(T))=\|p(B)\|=$ $\|p(T)\|$. If $\|p(A)\|>\|p(B)\|$, then we have $w(p(T)) \geq\|p(B)\|$ and $\|p(T)\|=$ $\|p(A)\|$. Since

$$
\|P(B)\|=\max _{|z| \leq r}|P(z)|=\max _{|z| \leq 1}\left|a_{0}+a_{1} r z+a_{2} r^{2} z^{2}+\cdots+a_{m} r^{m} z^{m}\right|,
$$

by the Carathéodory-Fejér Theorem (cf. $[9,2.5])$, we have

$$
\|p(B)\| \geq\left\|\left[\begin{array}{cc}
a_{0} & 0 \\
a_{1} r & a_{0}
\end{array}\right]\right\|=\left(\left|a_{0}\right|^{2}+\frac{r^{2}}{4}\left|a_{1}\right|^{2}\right)^{1 / 2}+\frac{r}{2}\left|a_{1}\right| .
$$

Therefore, we have

$$
\begin{aligned}
w(p(T)) & \geq\|p(B)\| \geq\left(\left|a_{0}\right|^{2}+\frac{r^{2}}{4}\left|a_{1}\right|^{2}\right)^{1 / 2}+\frac{r}{2}\left|a_{1}\right| \\
& \geq\left(r^{2}\left|a_{0}\right|^{2}+\frac{r^{2}}{4}\left|a_{1}\right|^{2}\right)^{1 / 2}+\frac{r}{2}\left|a_{1}\right| \\
& \geq r\left(\left(\left|a_{0}\right|^{2}+\frac{1}{4}\left|a_{1}\right|^{2}\right)^{1 / 2}+\frac{1}{2}\left|a_{1}\right|\right) \\
& =r\|p(A)\|=r\|p(T)\| .
\end{aligned}
$$

This implies that $n\left(\mathfrak{A}_{T}\right)=r$. This completes the proof.

Remark 1 . We recall that the numerical index of a normed algebra $\mathfrak{A}$ is defined by

$$
n(\mathfrak{A})=\inf \{v(a): a \in \mathfrak{A},\|a\|=1\},
$$

where $v(a)$ is the numerical radius of a (cf. [1, 2]). Then $e^{-1} \leq n(\mathfrak{A}) \leq 1$. In this case, the range of the numerical index is the whole of the closed interval $\left[e^{-1}, 1\right]$.

Remark 2. Take $T \in B(H)$ and consider the norm closed subalgebra $\mathfrak{A}_{T}$ of $B(H)$ generated by $T$ and $I$. Then we have an interest in the class of operators satisfying $n\left(\mathfrak{A}_{T}\right)=r$, in particular, $n\left(\mathfrak{A}_{T}\right)=1$. As in the proof of Theorem, $n\left(\mathfrak{A}_{T}\right)=1$ if and only if $\|p(T)\|=w(p(T))$ for every polynomial $p$. In [4, III], Fujii defined the notion of the class of operators, that is, an operator $T \in B(H)$ is hen-spectroid if $\tilde{\sigma}(T)$ is the spectral set for $T$, where $\tilde{\sigma}(T)$ is the complement of the unbounded component of the complement of the spectrum $\sigma(T)$ of $T$. By [4, III, Theorem 13], an operator $T \in B(H)$ satisfies $n\left(\mathfrak{A}_{T}\right)=1$ if and only if $T$ is hen-spectroid. 


\section{REFERENCES}

1. F. F. Bonsall and J. Duncan, Numerical ranges of operators on Normed spaces and of elements of Normed algebras, Cambridge Univ. Press, London and New York, 1971.

2. _ Numerical ranges. II, Cambridge Univ. Press, London and New York, 1973.

3. M. J. Crabb, J. Duncan, and C. M. McGregor, Characterizations of commutativity for $C^{*}$-algebras, Glasgow Math. J. 15 (1974), 172-175.

4. M. Fujii, On some examples of non-normal operators. I, Proc. Japan Acad. 47 (1971), 458-463; II, 48 (1972), 118-123; III, 48 (1972), 124-129.

5. U. Haagerup and P. de la Harpe, The numerical radius of a nilpotent on a Hilbert space, preprint, 1988.

6. P. R. Halmos, A Hilbert space problem book, 2nd ed., Springer-Verlag, Berlin and New York, 1982.

7. S. Hildebrandt, Über der numershen Wertebereich eines operators, Math. Ann. 163 (1966), 230-247.

8. T. Huruya, The normed space numerical index of $C^{*}$-algebras, Proc. Amer. Math. Soc. 63 (1977), 289-290.

9. M. Rosenblum and J. Rovnyak, Hardy classes and operator theory, Oxford Univ. Press, London and New York, 1985.

(Y. Nakamura) Division of Applied Mathematics, Research Institute of Applied ElecTRICITY, HOKKaIDo UNIVERSITY, SAPPORO, 060 JAPAN

(K.-S. Saito and K. Sakaba) Department of Mathematics, Faculty of Science, NiIgata UNIVERSITY, NiIGATA, 950-21 JAPAN

E-mail address: F00745@sinet.ad.jp 